\title{
Second-harmonic generation directionality is associated with neoadjuvant chemotherapy response in breast cancer core needle biopsies
}

Danielle E. Desa

Monisha Bhanote

Robert L. Hill

Joseph B. Majeski

Brandon Buscaglia

Marcus D'Aguiar

Robert Strawderman

David G. Hicks

Bradley M. Turner

Edward B. Brown 


\title{
Second-harmonic generation directionality is associated with neoadjuvant chemotherapy response in breast cancer core needle biopsies
}

\author{
Danielle E. Desa, ${ }^{a}$ Monisha Bhanote, ${ }^{b}$ Robert L. Hill, ${ }^{c}$ Joseph B. Majeski, ${ }^{\text {a }}$ Brandon Buscaglia, ${ }^{d}$ \\ Marcus D'Aguiar, ${ }^{d}$ Robert Strawderman, ${ }^{e}$ David G. Hicks, ${ }^{b}$ Bradley M. Turner, ${ }^{b}$ and Edward B. Brown ${ }^{a, *}$ \\ a University of Rochester, Hajim School of Engineering and Applied Sciences, Department of Biomedical Engineering, Rochester, New York, \\ United States \\ bUniversity of Rochester Medical Center, School of Medicine and Dentistry, Department of Pathology and Laboratory Medicine, Rochester, \\ New York, United States \\ cHarmonigenic Corporation, Rochester, New York, United States \\ ${ }^{d}$ Rochester Institute of Technology, Kate Gleason College of Engineering, Department of Biomedical Engineering, Rochester, New York, \\ United States \\ eUniversity of Rochester Medical Center, School of Medicine and Dentistry, Department of Biostatistics and Computational Biology, Rochester, \\ New York, United States
}

\begin{abstract}
Neoadjuvant chemotherapy (NACT) is routinely administered to subsets of breast cancer patients, including triple negative (TN) or human epidermal growth factor receptor 2-positive (HER2+) cancers. After NACT and subsequent surgical resection, $5 \%$ to $30 \%$ of patients have no residual invasive carcinoma, termed pathological complete response. Unfortunately, many patients experience little-to-no response after NACT and unnecessarily suffer its side effects. Methods are needed to predict an individual patient's response to NACT. Core needle biopsies, taken before NACT, consist of tumor cells and the surrounding extracellular matrix. We performed second-harmonic generation (SHG) imaging of fibrillar collagen in core needle biopsy sections as a possible predictor of response to NACT. The ratio of forward-to-backward scattering (F/B) SHG was assessed in the "tumor bulk" and "tumor-host interface" in HER2+ and TN core needle biopsy sections. Patient response was classified post-treatment using the Residual Cancer Burden (RCB) score. In HER2+ biopsies, RCB class was associated with F/B derived from the tumor-stromal interface, but not tumor bulk. F/B was not associated with $\mathrm{RCB}$ class in TN biopsies. These findings suggest that $\mathrm{F} / \mathrm{B}$ from needle biopsy sections may be a useful predictor of which patients will respond favorably to NACT, with the potential to help reduce overtreatment. ๑ The Authors. Published by SPIE under a Creative Commons Attribution 4.0 Unported License. Distribution or reproduction of this work in whole or in part requires full attribution of the original publication, including its DOI. [DOI: 10.1117/1.JBO.24.8.086503]
\end{abstract}

Keywords: neoadjuvant chemotherapy; breast cancer; second-harmonic generation; collagen; extracellular matrix; multiphoton laser scanning microscopy.

Paper 190104R received Apr. 8, 2019; accepted for publication Aug. 5, 2019; published online Aug. 27, 2019.

\section{Introduction}

Neoadjuvant chemotherapy (NACT) is a presurgical option for select breast cancer (BC) patients, including many triple-negative breast cancers (TNBCs) or human epidermal growth factor receptor 2-positive (HER2+) patients. NACT is recommended based on pathological assessment of an initial core needle biopsy, which is routinely taken for diagnosis prior to any therapeutic or surgical intervention. Core needle biopsies utilize $\mathrm{x}$-ray-guided stereotactic imaging, ultrasound guidance, or magnetic resonance imaging guidance to remove a sample of potentially diseased tissue that is then sectioned, stained, and used for diagnosis and determination of treatment options, including NACT. However, predicting who will and who will not benefit from NACT has proven to be challenging, exposing some patients to unnecessary toxicity and delaying administration of other life-saving treatments. ${ }^{1}$ Methods to stratify patients based on expected response to NACT are greatly desired.

Core needle biopsies typically consist of tumor cells as well as the surrounding extracellular matrix (ECM). A major

*Address all correspondence to Edward B. Brown, E-mail: Edward_Brown@ urmc.rochester.edu component of the ECM is fibrillar collagen, which is capable of producing an intrinsic optical signal called second-harmonic generation (SHG). SHG is a nonlinear optical phenomenon that occurs when two identical photons scatter off a noncentrosymmetric material to produce a single photon of exactly twice the energy of the incoming photons and hence half the wavelength. The main source of SHG in solid tumors is fibrillar collagen, and the directionality of SHG emission is sensitive to its microscopic arrangement including fibril diameter, spacing, and packing disorder within collagen fibers, which we collectively call the "microstructure."2-7 The ratio of forward-emitted (where "forward" is the direction of the excitation laser) to backwardemitted SHG, or F/B ratio, is sensitive to the microstructure, which is inherent to individual collagen fibers and is distinct from the overall arrangement of ensembles of fibers. ${ }^{8,9}$

SHG imaging has been used to study collagen remodeling in $\mathrm{BC}$ using a number of analysis methods. ${ }^{10-12}$ Provenzano et al. ${ }^{13}$ identified "tumor-associated collagen signatures" (TACS) by assessing fiber density and alignment relative to the tumor boundary during early disease, in-situ breast carcinomas, and invasive BC. Using a manual approach, TACS-3 (fiber alignment orthogonal to tumor boundary) was shown to be prognostic of disease-free survival by Conklin et al. ${ }^{9}$ Polarization-based 
SHG reflects the pitch angle of $\alpha$-helices within the collagen triple helix, which change with $\mathrm{BC}$ progression and has been applied to mouse models and human samples., ${ }^{2,14}$ Our group has previously shown that F/B measured from primary tumor samples is an independent prognostic indicator of the probability of remaining metastasis free for 10 years after tumor resection in a cohort of untreated estrogen receptor-positive (ER+), lymph node-negative (LNN) invasive ductal carcinoma (IDC) patients. ${ }^{15}$ Furthermore, we have shown that F/B from primary tumor samples is correlated with five-year survival in patients treated postoperatively with tamoxifen therapy. ${ }^{15}$

In this study, we assessed the relationship between Residual Cancer Burden (RCB) class, a measure of response to NACT, and collagen fiber orientation as well as microstructure (as reported by $\mathrm{F} / \mathrm{B}$ ) in different tumor regions of pre-NACT core needle biopsy sections from TNBC and HER2+ BC patients. We are interested in evaluating $\mathrm{F} / \mathrm{B}$ as a useful prognostic indicator of response to NACT; therefore, we measured F/B in samples that are currently in the clinical workflow, specifically formalinfixed, paraffin-embedded (FFPE) hematoxylin and eosin (H\&E)-stained sections from core needle biopsies. Hence, the $\mathrm{F} / \mathrm{B}$ we report here is not necessarily equal to $\mathrm{F} / \mathrm{B}$ that would be measured in unprocessed fresh biopsy tissue, because various steps in processing and mounting may affect the $\mathrm{F} / \mathrm{B}$ value.

\subsection{Clinical Background}

NACT is a well-established treatment option for the clinical management of patients with locally advanced BC (more than three positive lymph nodes or tumors $>5 \mathrm{~cm}$ ), patients who have medical contraindications to surgery at diagnosis but in whom surgery is anticipated at a later date (i.e., women diagnosed during late pregnancy), select cases of early stage BC patients in which breast-conserving surgery would be suboptimal, and select cases of TNBCs or HER2+ patients. NACT, which is administered preoperatively, is often used to downsize larger tumors, allowing breast-conserving surgery rather than mastectomy. ${ }^{1}$ Types of NACT include combinations of anthracyclines (e.g., doxorubicin or epirubicin) and taxanes (e.g., docetaxel or paclitaxel), with other drugs such as fluorouracil and cyclophosphamide. In HER2+ patients, targeted therapies with drugs such as trastuzumab, pertuzumab, lapatinib, and/ or bevacizumab are administered along with standard chemotherapy regimens, individualized to the patient. NACT has been shown to be as effective as adjuvant chemotherapy in terms of outcomes and is now considered as a possible standard option and a preferred approach for a select subset of BC patients. ${ }^{16}$ Multiple studies have shown that a patient's response to NACT is correlated with overall prognosis and disease-free survival. ${ }^{17}$ Specifically, patients who have a pathological complete response (pCR) after NACT exhibit better overall diseasefree survival and better long-term outcome than those with residual disease (RD). ${ }^{1,18-22}$ In particular, pCR is most strongly correlated with better overall disease-free survival and better long-term outcome in TNBC and HER2+ BC patients. ${ }^{1,18,23,24}$ However, NACT has unpleasant and debilitating side effects, including, but not limited to, nausea, vomiting, fatigue, hair loss, infection, and mouth sores. Bone marrow reserves and renal function decrease with age, increasing the probability of myelosuppression, cardiodepression, peripheral neuropathy, and neurotoxicity. ${ }^{25}$ Further, NACT has been shown to increase the density of tumor microenvironment of metastasis (TMEM) complexes in human breast tumors. TMEM complexes are thought to be sites of tumor cell intravasation and their density is correlated with metastatic outcome in human mammary carcinomas. ${ }^{26}$ For these reasons, there is an identified pressing need to administer NACT only to those who will benefit from it and not administer it to those who will not benefit. ${ }^{19}$

Though there are different definitions of pCR, many studies concede that true pCR includes a lack of residual invasive disease in both the breast and axillary lymph nodes. ${ }^{18,27-30} \mathrm{RCB}$, one method of quantifying $\mathrm{RD}$ in a resected specimen, is a score calculated based on the presence or absence of invasive tumor in the primary tumor bed, and presence or absence of tumor in the lymph nodes..$^{20,21,31,32}$ Patients are sorted into one of four classes based on their RCB scores, where RCB-0 corresponds to pCR, RCB-I and RCB-II represent intermediate outcomes, and RCBIII indicates extensive RD. ${ }^{31}$ In numerous clinical trials and meta-analyses, RCB class has a significant positive correlation with overall survival and discriminates between patients with favorable and unfavorable prognoses. ${ }^{21}$ Specifically, RCB-0 and RCB-I have been shown to have the same positive five-year prognosis no matter the NACT, adjuvant chemotherapy, or pathological stage, while RCB-II and RCB-III have worse prognoses. ${ }^{31}$ To this end, an improvement in the ability to identify those $\mathrm{BC}$ patients who are most likely to have a class of RCB-0 or RCB-I, and therefore a better response to NACT, will assist decision-making regarding surgery, adjuvant chemotherapy, and follow-up care. ${ }^{19}$

\section{Methods}

\subsection{Patient Samples}

A total of 29 patients with HER2+ biopsy specimens and 27 patients with TNBC biopsy specimens, all with available HER2 and RCB results, were identified from the pathology files at the University of Rochester Medical Center (URMC) (2009 to 2019). Use of patient samples was approved by the Institutional Review Board at URMC. All TNBC patients received some type of NACT regimen which included standard combinations of a taxane, an anthracycline, cyclophosphamide, etoposide, 5-fluorouracil, methotrexate, and/or platinum therapy. All HER2+ patients received trastuzumab and/or pertuzumab with some type of NACT regimen which included standard combinations of a taxane, an anthracycline, cyclophosphamide, and/or platinum therapy. All biopsy samples collected prior to NACT administration were processed in the URMC pathology laboratory and mounted on slides as 5- $\mu$ m-thick FFPE sections, prior to routine H\&E staining. After NACT administration and tumor resection, all patients received either partial or total mastectomy with appropriate surgical evaluation of lymph nodes. Postmastectomy tissues were processed in the URMC pathology laboratory as 5- $\mu$ m-thick FFPE sections and were H\&E stained. All tumor H\&E slides and immunohistochemistry stains were reviewed by at least two board-certified breast pathologists with manual interpretation of HER2 (rabbit antihuman HER2, Dako HercepTest ${ }^{\mathrm{TM}}$ ). Fluorescence in-situ hybridization (FISH) was performed on all equivocal HER2 immunohistochemistry results (HER2 IQFISH pharmDx, FDA kit, Dako), and the FISH results were used in lieu of the immunohistochemistry for these cases.

The evaluation of the extent of RD following neoadjuvant therapy was performed on the post-treatment pathology material using the published method of Symmans et al..$^{21,31,32}$ and the associated RCB online calculator. Briefly, the gross description 
along with clinical imaging studies and specimen photographs were used to determine the two largest dimensions of the residual primary tumor bed in the resection specimen. Evaluation of microscopic sections was used to determine (1) the proportion of the primary tumor bed that contained metastatic carcinoma, (2) the number of axillary lymph nodes that contained metastatic carcinoma, and (3) the diameter of the largest metastatic deposit. This information was entered into the online calculator and the RCB score and RCB class were recorded.

\subsection{Imaging}

A Spectra Physics MaiTai Ti:sapphire laser (circularly polarized at the sample using a Berek Compensator, $100 \mathrm{fs}$ pulses at $80 \mathrm{MHz}, 810 \mathrm{~nm}, \sim 3 \mathrm{~mW}$ at the sample) was directed through an Olympus Fluoview FV300 scanner. The laser was focused through an Olympus UMPLFL20XW water-immersion lens (20×, 0.95 NA), which subsequently captured backwardpropagating SHG signal. This SHG signal was separated from the excitation beam using a 670-nm dichroic mirror, filtered (HQ405/30 m-2P, Chroma, Rockingham, Vermont), and collected by a photomultiplier tube (Hamamatsu HC125-02). The forward-scattered SHG was collected through an Olympus 0.9 NA condenser, reflected by a $565-\mathrm{nm}$ dichroic mirror (565 DCSX, Chroma, Rockingham, Vermont) to remove excitation light, filtered (HQ405/30 m-2P, Chroma, Rockingham, Vermont) and captured by an identical photomultiplier tube (Hamamatsu HC125-02). Quantification of " $F$ " and "B" signals while scanning the excitation wavelength from 740 to $880 \mathrm{~nm}$ (i.e., just below to just above the emission filter bandpass) enabled us to estimate the extent of contamination of the SHG signal with two-photon-excited fluorescence, revealing that $\sim 1 \%$ of the detected forward-scattered photons and $\sim 10 \%$ of backward-scattered photons were from two-photon-excited fluorescence.
A microscopist who was blinded to the patient outcome (i.e., eventual RCB class) performed the SHG imaging and analysis. Three fields of view (FOVs) were taken in the "tumor bulk" (solid box, Fig. 1) of each 5- $\mu$ m-thick pretreatment core needle biopsy sample. The "tumor-host interface" was defined as the stroma immediately surrounding the tumor bulk. Three FOVs were also taken in the tumor-host interface, with the tumor bulk just out of the FOV (dashed box, Fig. 1). Forward- and backward-scattered SHG images were collected simultaneously. Examples of the H\&E-stained FOV and corresponding SHG images are shown in Fig. 2. Images from these core needle biopsy sections revealed a random network of collagen fibers often encapsulating SHG-dark nodules containing highly cellular areas, consistent with our previous images of primary tumor sections. ${ }^{15,33}$ Interestingly, close inspection of forward $(\mathrm{F})$ and backward (B) images often revealed the characteristic "segmented" appearance of fibrils in the B image relative to the F image (Fig. 2), as previously reported in other tissue types., ${ }^{4,8}$

\subsection{Image Analysis: Intensity Mask-Based F/B}

A single value of $\mathrm{F} / \mathrm{B}$ for a $\mathrm{FOV}$ was derived from the $\mathrm{F}$ and $\mathrm{B}$ images of that FOV using Image $\mathrm{J}^{34,35}$ as previously described. ${ }^{15,33}$ Briefly, an $\mathrm{F}$ image of forward-propagating SHG and a B image of backward-propagating SHG were collected in each FOV. All images were background-subtracted and a pair of masks was created to distinguish background pixels from collagen fibers. To produce the masks (one mask for the $\mathrm{F}$ image and one for the $\mathrm{B}$ image), a blinded user selected a threshold to best distinguish pixels within fibers from background pixels. The same two thresholds were used for all $\mathrm{F}$ and all $\mathrm{B}$ images in a single imaging session. All pixels above the lower threshold were set to 1 and those below the lower threshold to 0 , producing binary $\mathrm{F}$ and $\mathrm{B}$ masks. These binary $\mathrm{F}$ and $\mathrm{B}$ masks were multiplied together to create a final mask of pixels within fibers. The background-subtracted $\mathrm{F}$ and $\mathrm{B}$ images were

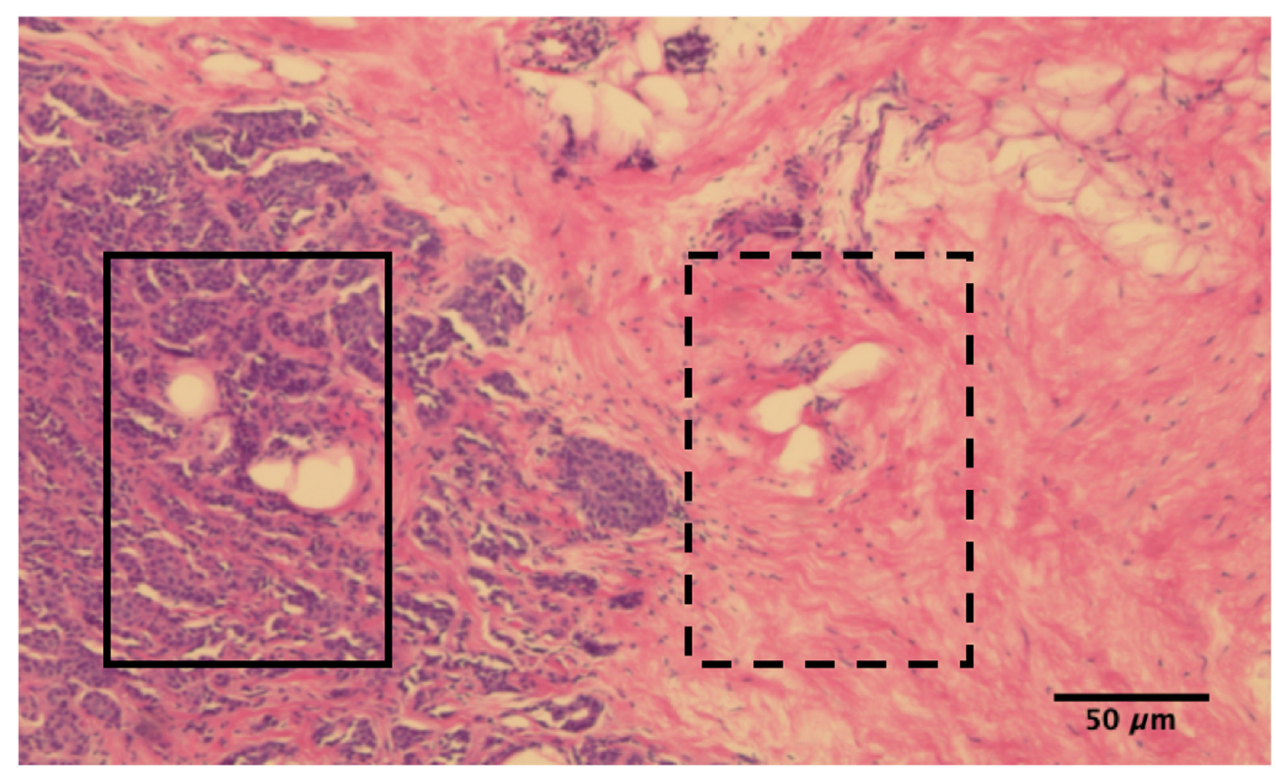

Fig. 1 Core needle biopsy sections contain tumor cells and surrounding stroma. Pretreatment core needle biopsy sections contain heterogeneous cellular and noncellular regions. We have defined both the tumor bulk (solid box), which consists primarily of tumor cell clusters, and the tumor-host interface (dashed box), the matrix that surrounds the cell clusters and consists mainly of collagen and structural proteins. Three images were taken in the tumor bulk and three in the tumor-host interface for each H\&E-stained core needle biopsy. 


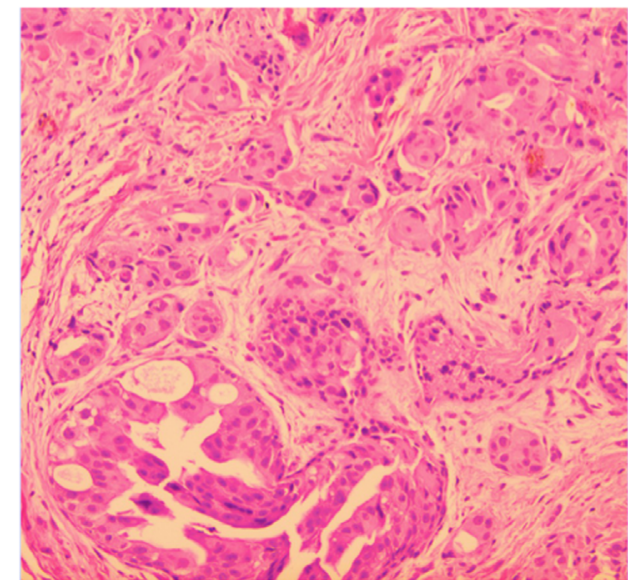

(a)

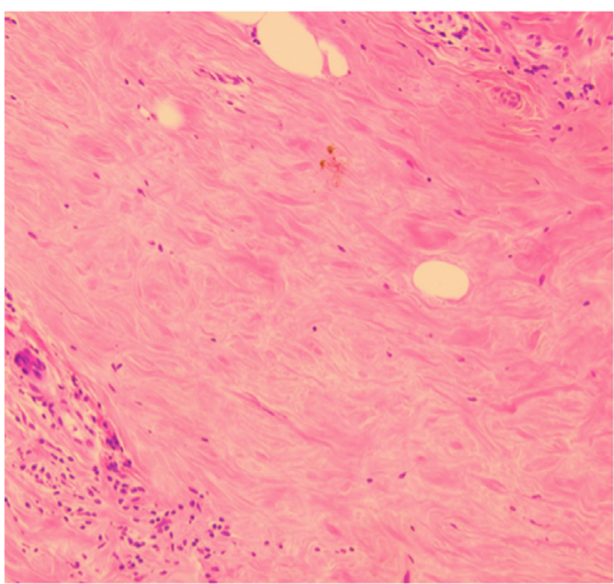

(c)

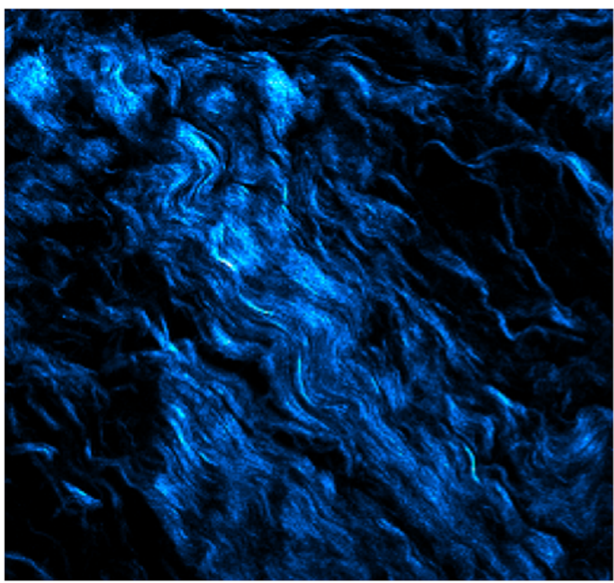

(e)

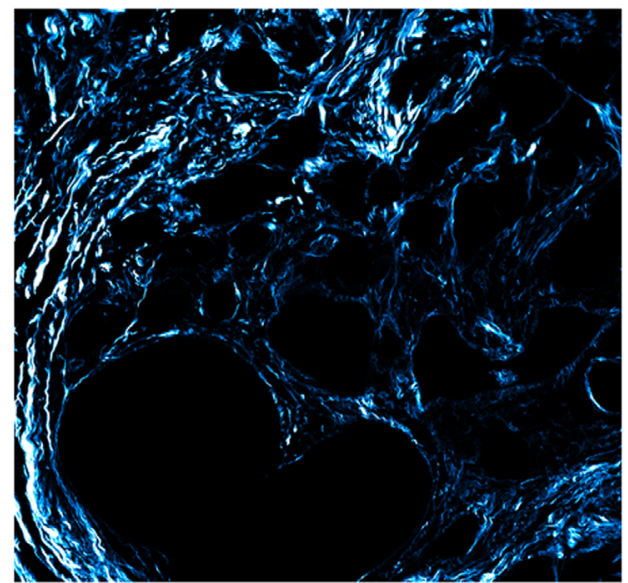

(b)

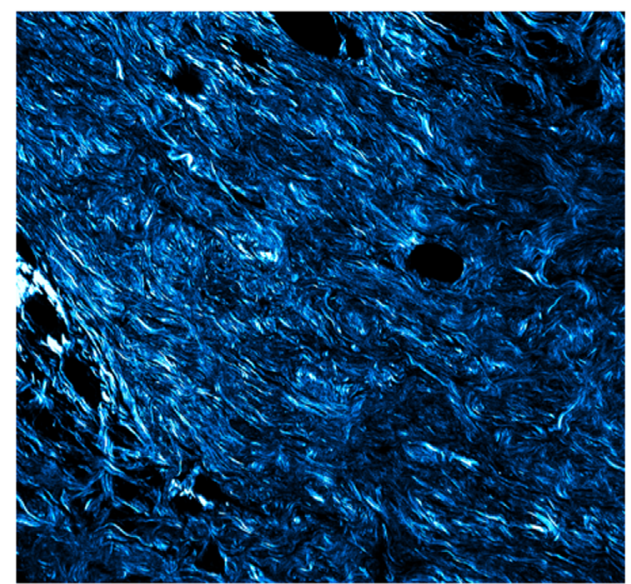

(d)

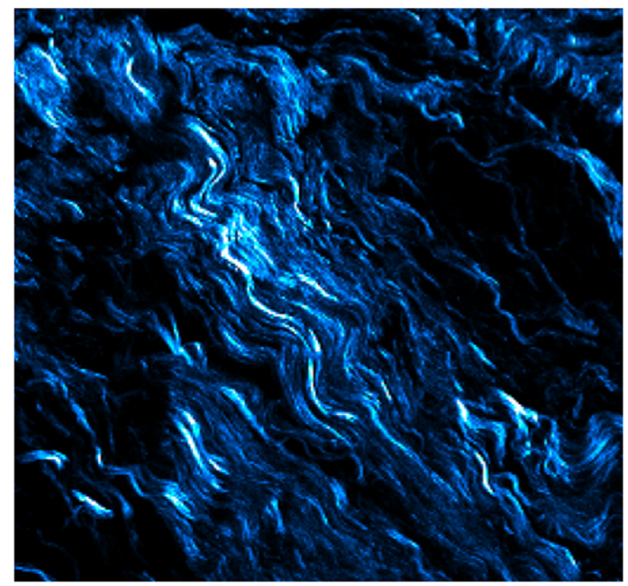

(f)

Fig. 2 Tumor bulk and tumor-host interface can be imaged with SHG microscopy. Core needle biopsy sections contain the bulk of the tumor and the tumor-host interface consisting mostly of collagen. Here, the same region of the tumor bulk is shown: (a) transmitted light microscopy (H\&E stained) and (b) twophoton forward-scattered SHG image. In the same core, a FOV containing the tumor-host interface is shown: (c) transmitted light microscopy (H\&E stained) and (d) two-photon forward-scattered SHG image of the same region. Note: (a)-(d) are $\sim 660 \mu \mathrm{m}$ across. (e) Backward-scattered and (f) forward-scattered SHG images reveal the fibrillar collagen network, often encapsulating SHG-dark cellular areas. Characteristic segmented fibrils appear in (e) the backward-scattered image, relative to (f) the corresponding forward-scattered image. Note: (e) and (f) are $\sim 420 \mu \mathrm{m}$ across. 
divided to produce a single F/B image, which was multiplied by this final mask. The average value of the nonzero pixels from the resultant image yielded the average F/B of the entire FOV. The F/B values from the three FOVs in each location type were averaged to provide a single $\mathrm{F} / \mathrm{B}$ value from the tumor bulk and one from the tumor-host interface for each patient.

\subsection{Image Analysis: Coherency Mask-Based F/B}

In addition to the selection of pixels based on an intensity threshold, we also investigated the selection of pixels based on their grouping into linear structures, as quantified by pixel "energy" and "coherency," as previously described. ${ }^{36}$ SHG images were background-subtracted and day-to-day variations in intensity were normalized, as described above. The opensource toolbox OrientationJ was used to select those pixels most relevant in this analysis. This toolbox enables the production of binary image masks where the pixels that are rejected or passed through the mask are selected based on their energy and/or coherency contribution. For this analysis, pixels passed by the mask were chosen to have a minimum of $2 \%$ of both normalized energy and coherency, ${ }^{37}$ where the energy and coherency values are calculated from a $2 \times 2$ structure tensor that is determined for each pixel. The structure tensor is defined as

$$
J=\left[\begin{array}{ll}
\left\langle f_{x}, f_{x}\right\rangle & \left\langle f_{x}, f_{y}\right\rangle \\
\left\langle f_{x}, f_{y}\right\rangle & \left\langle f_{y}, f_{y}\right\rangle
\end{array}\right],
$$

where $f_{x}$ and $f_{y}$ are the partial derivatives of the intensity in the $x$ and $y$ directions, respectively. ${ }^{37}$ This tensor is found for each pixel by using a cubic B-spline interpolation in both the $x$ and $y$ directions to find the continuous spatial derivative. Once the tensor values are known, the coherency is calculated based on the eigenvalues as

$$
\text { coherency }=\frac{\lambda_{\max }-\lambda_{\min }}{\lambda_{\max }+\lambda_{\min }},
$$

where $\lambda_{\max }$ is the largest eigenvalue and $\lambda_{\min }$ is the smallest. Then the energy of the tensor is found as the trace of the structure tensor or

$$
\text { energy }=\left\langle f_{x}, f_{x}\right\rangle+\left\langle f_{y}, f_{y}\right\rangle \text {. }
$$

Once the binary image masks were obtained for both the forward and backward images, they were multiplied together to produce a final mask. The background-subtracted forward and backward images were divided to produce a raw F/B image, which was multiplied by the final mask. All pixels of value zero were excluded, and the average of this image gave an average F/B for a particular FOV.

\subsection{Image Analysis: Collagen Fiber Angle at Tumor Boundary}

In addition to measuring $\mathrm{F} / \mathrm{B}$ in each tumor region, we assessed the organization of collagen fibers at the tumor-host interface. Using the method of Provenzano et al., ${ }^{13}$ the boundary between the tumor bulk and the interface was defined and then the angle of collagen fibers relative to the tangent of the boundary was measured every $30 \mu \mathrm{m}$. This analysis was performed in three imaged locations per biopsy section.

\subsection{Calibration}

For each imaging session, a background image of no sample and a reference $\mathrm{F}$ and $\mathrm{B}$ image of a dilute stock solution of isotropically emitting fluorescein isothiocyanate (FITC) were collected (to quantify day-to-day variations in sample alignment). Day-today variations in system alignment were normalized by dividing each $\mathrm{F} / \mathrm{B}$ value by the $\mathrm{F} / \mathrm{B}$ value measured for the FITC stock solution (expected $\mathrm{F} / \mathrm{B}=1$ ) during that imaging session.

\subsection{Statistical Analysis}

Logistic regression was used to assess the association between $\mathrm{F} / \mathrm{B}$ and binary response variable "RCB class $>1$." (This is a variable that can be either 0 or 1 . A value of " 0 " is assigned when RCB class is 0 or I, and a value of " 1 " if RCB class is II or III.) A likelihood ratio test was used to calculate the $p$-value for the test of whether the regression coefficient for F/B was zero. All graphs were generated using GraphPad Prism 8 and statistics were assessed using JMP Pro 14.

\section{Results}

We have previously shown that F/B from SHG images is prognostic of metastasis-free survival in a cohort of untreated ER+ LNN IDC patients. Further, a subset of these patients was administered postoperative (tamoxifen) therapy after they were diagnosed with distant recurrence and $\mathrm{F} / \mathrm{B}$ of the primary tumor was correlated with five-year survival in these patients. ${ }^{15}$ As a result, we explored the relationship between $\mathrm{F} / \mathrm{B}$ in core needle biopsy samples and a patient's subsequent response to NACT. The goal of this study was to assess a potential relationship between $\mathrm{F} / \mathrm{B}$ derived from different regions of pretreatment biopsy sections and NACT response. As RCB accounts for lymph node involvement as well as the primary tumor bed, it was used as an assessment of patient response to NACT. RCB-0 and RCB-I have been shown to have the same five-year prognosis no matter the neoadjuvant therapy, adjuvant therapy, or pathologic stage; therefore, RCB-0/I and II/III were grouped together for our comparison. ${ }^{31}$ We performed SHG imaging on pretreatment biopsy sections to see if we could distinguish patients who responded favorably to NACT from those with RD.

\subsection{HER2+ Samples: Intensity Masks}

When F/B is calculated from SHG images using masks that select collagen pixels and reject background based on pixel intensity, the average F/B from the tumor bulk was significantly different from that of the tumor-host interface $[\mathrm{F} / \mathrm{B}=8.84 \pm 0.67$ (tumor bulk) or $15.35 \pm 1.01$ (tumor-host interface), $p<0.0001$, data not shown]. Intensity mask-based $\mathrm{F} / \mathrm{B}$ in each region type was subsequently stratified by the patient's RCB class post-NACT after mastectomy. Samples were grouped as RCB-0/I or RCB-II/III in both the tumor bulk $(\mathrm{F} / \mathrm{B}=8.87 \pm 0.88$ or $8.78 \pm 1.05$, respectively) and the tumor-host interface $(\mathrm{F} / \mathrm{B}=16.51 \pm 0.95$ or $12.32 \pm 1.84$, respectively). When coded as a binary response (0/I or II/III), intensity mask-based F/B derived from the tumor-host interface was associated with RCB class ( $p=0.0246$; Fig. 3 ). However, intensity mask-based F/B was not associated with RCB class when derived from images of the tumor bulk $(p=0.297$; Fig. 3). 

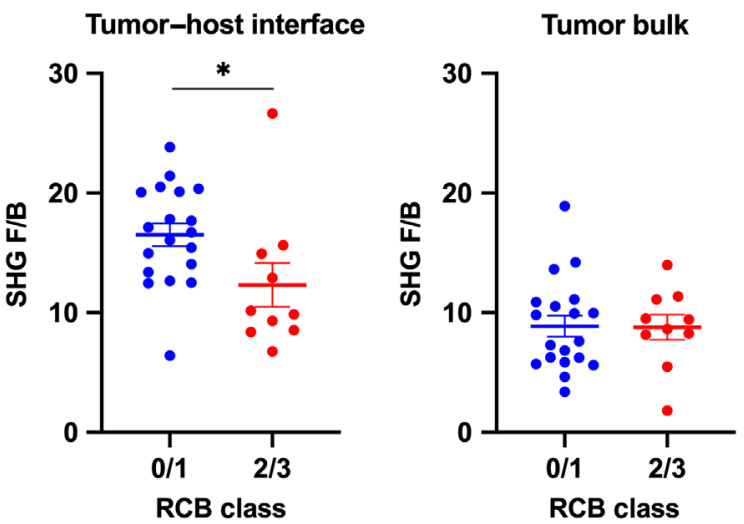

Fig. 3 Intensity threshold-based F/B is correlated with RCB class in HER2+ biopsy samples. Intensity threshold-based F/B was calculated from SHG images of the tumor bulk and tumor-host interface in each HER2+ core needle biopsy $(n=29)$. Each point is the average of three tumor bulk or tumor-host interface FOVs. Two categories of NACT response were determined using RCB class, grouped as RCB-O/I and RCB-II/III. F/B is correlated with RCB class in the tumor-host interface, but not in the bulk of the tumor. Error bars $=$ standard error of the mean (SEM), $p=0.0246$.

\subsection{HER2+ Samples: Coherency Masks}

The F/B was then derived using masks that select collagen pixels and reject background pixels based on their residence in linear structures (i.e., coherency). In each region type (tumor bulk and tumor-host interface), the F/B was coded as a binary response $(0 / \mathrm{I}$ or II/III) post-NACT after mastectomy. Samples were again grouped as RCB-0/I or II/III in the tumor bulk $(\mathrm{F} / \mathrm{B}=33.03 \pm 2.73$ or $35.12 \pm 3.76$, respectively $)$ and tumorhost interface $(\mathrm{F} / \mathrm{B}=39.47 \pm 2.25$ or $35.66 \pm 3.85$, respectively). Coherency mask-based $\mathrm{F} / \mathrm{B}$ was not associated with RCB class when measured either in the tumor-host interface or tumor bulk ( $p=0.643$ and 0.346 , respectively; Fig. 4).

\subsection{TNBC Samples: Intensity Masks}

In TNBC needle biopsy samples, the average intensity maskbased F/B derived from the tumor bulk was significantly different from that of the tumor-host interface $[\mathrm{F} / \mathrm{B}=7.48 \pm 0.55$
Tumor-host interface

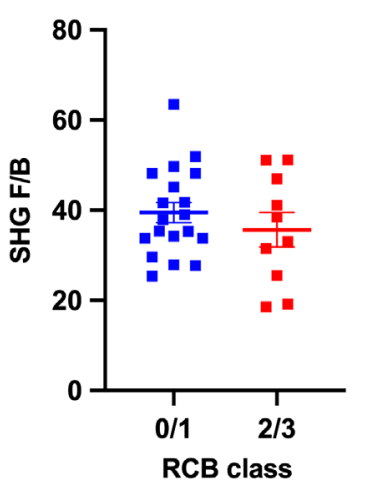

Tumor bulk

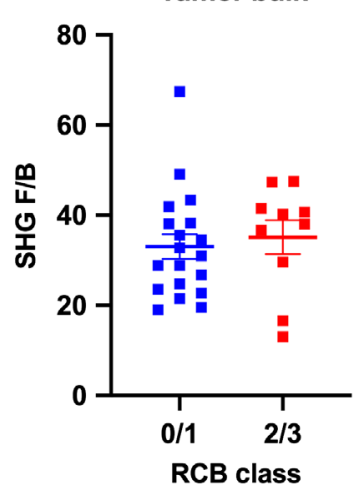

Fig. 4 Coherency-based F/B is not correlated with RCB class in HER2+ biopsy samples. Coherency-based F/B was calculated from tumor bulk and tumor-host interface in each HER2+ core needle biopsy $(n=29)$ using OrientationJ. Coherency-based F/B is not correlated with RCB class in either region of HER2+ biopsy samples. Error bars $=$ SEM.
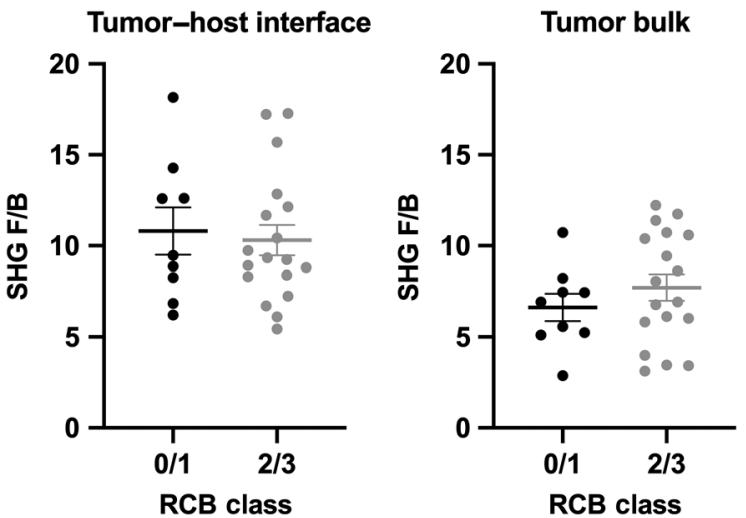

Fig. 5 Intensity threshold-based F/B is not correlated with RCB class in TNBC biopsy samples. Intensity threshold-based F/B was calculated from both tumor bulk and tumor-host interface in each TNBC core needle biopsy $(n=27)$. F/B is not correlated with RCB class in either region of TNBC biopsy samples. Error bars $=$ SEM.

(tumor bulk) or $10.67 \pm 0.69$ (tumor-host interface), $p=$ 0.0008 , data not shown]. The $\mathrm{F} / \mathrm{B}$ in each region was coded as a binary response according to a patient's RCB class postNACT after mastectomy. Intensity mask-based F/B was not associated with RCB class $0 / \mathrm{I}$ or II/III in the tumor bulk $(6.62 \pm 0.75$ or $7.72 \pm 0.72$, respectively; $p=0.329$; Fig. 5). Intensity mask-based F/B was also not associated with RCB class $0 / \mathrm{I}$ or II/III in the tumor-host interface $(10.81 \pm 3.89$ or $10.31 \pm 3.56$, respectively; $p=0.73$; Fig. 5).

\subsection{TNBC Samples: Coherency Masks}

Coherency mask-based F/B in each region type was coded as a binary response by RCB class post-NACT after mastectomy. Samples were again grouped as RCB-0/I or II/III in the tumor bulk $(\mathrm{F} / \mathrm{B}=29.98 \pm 4.20$ or $34.18 \pm 2.98$, respectively $)$ and tumor-host interface $(\mathrm{F} / \mathrm{B}=35.99 \pm 3.81$ or $35.23 \pm 2.57$, respectively). Coherency mask-based $\mathrm{F} / \mathrm{B}$ was not associated with RCB class when derived from either the tumor-host interface or tumor bulk ( $p=0.400$ and 0.862, respectively; Fig. 6).
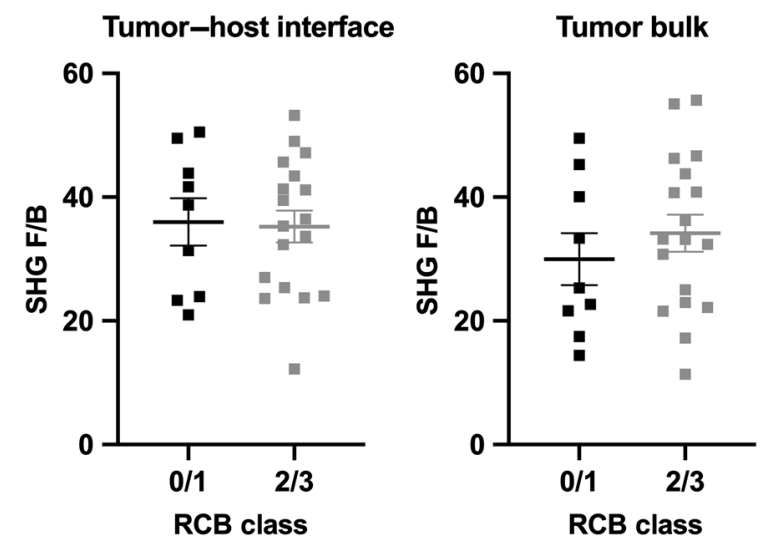

Fig. 6 Coherency-based F/B is not correlated with $R C B$ class in TNBC biopsy samples. Coherency-based F/B was calculated from tumor bulk and tumor-host interface in each TNBC core needle biopsy ( $n=27)$ using OrientationJ, as previously described. Coherencybased F/B is not correlated with RCB class in either region of TNBC biopsy samples. Error bars $=$ SEM. 

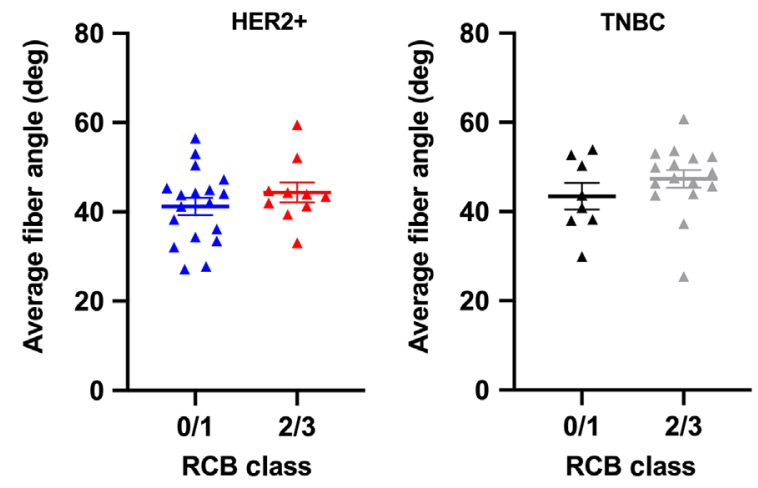

Fig. 7 Average fiber angle at the tumor boundary is not correlated with RCB class in HER2+ or TNBC biopsy samples. The collagen fiber angle relative to the tumor boundary was measured every $30 \mu \mathrm{m}$ in HER2+ $(n=28)$ and TNBC core needle biopsies $(n=24)$. The average fiber angle of each sample is not correlated with RCB class. Error bars $=$ SEM.

\subsection{HER2+ and TNBC Samples: Fiber Angle at Tumor Boundary}

The average collagen fiber angle along the tumor boundary for each patient was coded as a binary response by RCB class postNACT after mastectomy. Samples were again grouped as RCB0/I or II/III in both HER2+ and TNBC patient groups. Average fiber angle was not associated with RCB class when measured in either HER2+ $(p=0.298)$ or TNBC samples $(p=0.258$; Fig. 7).

\section{Discussion}

NACT has become a recommended treatment option for certain patients with locally advanced or inoperative BC. However, a significant number of patients do not respond to NACT and would instead benefit from immediate tumor resection and other therapies. The ability to predict patient response to NACT would greatly reduce overtreatment, mitigate side effects and cost, and optimize individual patient treatment regimens.

Solid tumors are heterogeneous, consisting of tumor cells as well as soluble growth factors, stromal cells, and the ECM. ${ }^{38}$ The ECM is dynamic, responding to and influencing tumor cell behavior, and this relationship is integral to tumor chemoresistance and metastasis. ${ }^{38-42}$ Accordingly, we investigated the tumor ECM as a candidate for predicting NACT response in two subsets of BC patients that typically receive it-TNBC and HER2+ $\mathrm{BC}$ patients. We have previously shown that the $\mathrm{F} / \mathrm{B}$ derived from SHG images is associated with metastasis-free survival in a subset of untreated IDC patients and progression-free survival in IDC patients treated with tamoxifen after a distant recurrence was diagnosed. ${ }^{15}$ Therefore, we used SHG imaging and $\mathrm{F} / \mathrm{B}$ measurements to study the ECM and subsequent patient response to NACT.

We imaged core needle biopsy sections taken from IDC patients prior to NACT administration and assessed the relationship between SHG F/B and post-treatment RCB class. This technique is nondestructive, rapid, and fits into the typical pathology workflow, as imaging can be performed on the same sections used for diagnosis without additional staining or tissue processing. SHG imaging complements genetically based diagnostic tests by additionally assessing the ECM surrounding tumor cells. We first analyzed SHG F/B from pixels above a threshold brightness that served to distinguish pixels in collagen fibers from background pixels. For comparison, we also assessed F/B using a method to identify pixels for analysis based on their grouping into linear arrangements. We found that intensity mask-based $\mathrm{F} / \mathrm{B}$ was associated with NACT response in HER2+ BC patients, but not TNBC patients, while coherency mask-based F/B was not associated with NACT response in either patient group. Further, there was no association between NACT response and the average angle of collagen fibers relative to the tumor boundary. This suggests that the relationship between F/B and response to NACT may lie in the collagen microstructure (fibril properties) rather than the overall fiber alignment. It is worth noting that this statistically significant relationship was derived from images taken in the collagenous tumor-host interface rather than the tumor bulk (Fig. 3). This suggests the possibility that chemoresistant cells occupy the tumor-host interface rather than residing in the bulk of the tumor. Future improvements in the ability of $\mathrm{F} / \mathrm{B}$ to predict RCB status might be found by more efficiently separating the small contaminating fluorescence signal from SHG (by using narrower bandpass filters, time-domain imaging, as well as exploring other excitation wavelengths), depositing tissue sections on antireflection-coated slides to more accurately measure the true $\mathrm{F} / \mathrm{B}$, or even assessing $\mathrm{F} / \mathrm{B}$ from fresh tissue if that proves more predictive. However, any methodological modifications that would require alteration of the current clinical workflow should be carefully considered, as they may hinder adoption of any resultant predictive method.

Several possible mechanisms of chemoresistance may be responsible for the relationship between F/B and the outcomes we observed in the HER2+ population. Systemic drug delivery is controlled by vascular transport (flux via the vascular network), transvascular transport (flux through vessel walls and basement membranes), and interstitial transport (diffusion and convection through a tumor to target cells). ${ }^{43}$ The ECM affects each of these transport mechanisms directly and/or indirectly and can be studied using multiphoton microscopy-based methods. ${ }^{43-47}$ Increased production of ECM components such as hyaluronan and collagen causes solid stress, constricting vasculature and normal cellular function. In addition, leaky and convoluted vessels combined with reduced interstitial pressure further hinder drug delivery via blood flow and diffusion. ${ }^{48}$ Finally, collagen orientation and organization influence drug diffusion rates through the ECM by steric hindrance. ${ }^{49-51}$ In addition to affecting chemotherapy efficacy by influencing transport of drugs to target cells, collagen organization can influence the resistance of cells to that drug. This can occur via interactions between collagen and cancer cell receptors and activation of downstream signaling pathways. ${ }^{38,48}$

\section{Conclusions}

NACT is often used to reduce tumors prior to operation in $\mathrm{BC}$ patients. The pCR to NACT is considered to be a positive prognostic indicator and is correlated with a good long-term outcome, particularly in HER2+ and TNBC patients. The RCB is a method of scoring a patient's response to NACT based on the amount of residual invasive carcinoma in the primary tumor bed and the presence or absence of invasive carcinoma in the lymph nodes. SHG imaging is a nonlinear optical method that utilizes intrinsic properties of fibrillar collagen in solid tumor ECM. Directional (F/B) scattering from fibrillar collagen is sensitive to microstructure properties, including fibril diameter, packing disorder, and spacing, and has been correlated with 
metastasis-free survival response in a subset of IDC patients. SHG imaging was performed on two subsets of core needle biopsy sections taken from HER2+ and TNBC patients prior to NACT administration and microstructure analyzed two ways. Intensity mask-based F/B derived from the tumor-host interface, but not the tumor bulk, was associated with post-treatment RCB class in HER2+ biopsies. There was no association between coherency mask-based F/B and RCB class in HER2+ samples. In triple-negative biopsies, no association was found between F/B determined using intensity or coherency-based methods in either the tumor bulk or the tumor-host interface. The average fiber orientation relative to the tumor boundary was not correlated with RCB class in either HER2+ or TNBC patients. This pilot study suggests that intensity mask-based F/B may be a useful predictor of response to NACT in HER2+ patients and warrants further investigation. Development of a convenient F/B-based method to predict which patients will respond favorably to NACT will help to inform clinical decision-making for $\mathrm{BC}$ patients and their healthcare providers.

\section{Disclosures}

R.L.H., E.B.B., D.G.H., B.M.T., B.B., and D.E.D. are inventors on a provisional patent related to the methods used in the paper. All other authors declare that they have no competing interests.

\section{Acknowledgments}

Funding for this research was provided by the Wilmot Cancer Institute at the University of Rochester Medical Center and the Breast Cancer Coalition of Rochester (DED), as well as the Department of Defense Breast Cancer Research Program (BCRP) W81XWH-17-1-0011 (EBB).

\section{References}

1. X. B. Li et al., "Biomarkers predicting pathologic complete response to neoadjuvant chemotherapy in breast cancer," Am. J. Clin. Pathol. 145(6), 871-878 (2016).

2. X. Han et al., "Second harmonic properties of tumor collagen: determining the structural relationship between reactive stroma and healthy stroma," Opt. Express 16(3), 1846-1859 (2008).

3. R. M. Williams, W. R. Zipfel, and W. W. Webb, "Interpreting secondharmonic generation images of collagen I fibrils," Biophys. J. 88(2), 1377-1386 (2005).

4. R. Lacomb et al., "Phase matching considerations in second harmonic generation from tissues: effects on emission directionality, conversion efficiency and observed morphology," Opt. Commun. 281(7), 18231832 (2008).

5. O. Nadiarnykh et al., "Alterations of the extracellular matrix in ovarian cancer studied by second harmonic generation imaging microscopy," BMC Cancer 10, 94 (2010).

6. V. Ajeti et al., "Structural changes in mixed Col I/Col V collagen gels probed by SHG microscopy: implications for probing stromal alterations in human breast cancer," Biomed. Opt. Express 2(8), 2307-2316 (2011).

7. K. R. Campbell et al., "Second-harmonic generation microscopy analysis reveals proteoglycan decorin is necessary for proper collagen organization in prostate," J. Biomed. Opt. 24(6), 066501 (2019).

8. R. A. Rao, M. R. Mehta, and K. C. Toussaint Jr., "Fourier transformsecond-harmonic generation imaging of biological tissues," Opt. Express 17(17), 14534-14542 (2009).

9. M. W. Conklin et al., "Aligned collagen is a prognostic signature for survival in human breast carcinoma," Am. J. Pathol. 178(3), 1221-1232 (2011).
10. R. A. Natal et al., "Collagen analysis by second-harmonic generation microscopy predicts outcome of luminal breast cancer," Tumour Biol. 40(4) (2018).

11. J. S. Bredfeldt et al., "Automated quantification of aligned collagen for human breast carcinoma prognosis," J. Pathol. Inf. 5(1), 28 (2014).

12. G. Falzon, S. Pearson, and R. Murison, "Analysis of collagen fibre shape changes in breast cancer," Phys. Med. Biol. 53(23), 6641-6652 (2008).

13. P. P. Provenzano et al., "Collagen reorganization at the tumor-stromal interface facilitates local invasion," BMC Med. 4(1), 38 (2006).

14. R. Ambekar et al., "Quantifying collagen structure in breast biopsies using second-harmonic generation imaging," Biomed. Opt. Express 3(9), 2021-2035 (2012).

15. K. Burke et al., "Using second harmonic generation to predict patient outcome in solid tumors," BMC Cancer 15, 929 (2015).

16. L. N. Chaudhary, K. H. Wilkinson, and A. Kong, "Triple-negative breast cancer: who should receive neoadjuvant chemotherapy?" Surg. Oncol. Clin. N. Am. 27(1), 141-153 (2018).

17. G. von Minckwitz et al., "Response-guided neoadjuvant chemotherapy for breast cancer," J. Clin. Oncol. 31(29), 3623-3630 (2013).

18. G. von Minckwitz et al., "Definition and impact of pathologic complete response on prognosis after neoadjuvant chemotherapy in various intrinsic breast cancer subtypes," J. Clin. Oncol. 30(15), 1796-1804 (2012).

19. M. Kaufmann et al., "Recommendations from an international expert panel on the use of neoadjuvant (primary) systemic treatment of operable breast cancer: new perspectives 2006," Ann. Oncol. 18(12), 1927-1934 (2007).

20. E. Provenzano et al., "Standardization of pathologic evaluation and reporting of postneoadjuvant specimens in clinical trials of breast cancer: recommendations from an international working group," Mod. Pathol. 28(9), 1185-1201 (2015).

21. W. F. Symmans et al., "Long-term prognostic risk after neoadjuvant chemotherapy associated with residual cancer burden and breast cancer subtype," J. Clin. Oncol. 35(10), 1049-1060 (2017).

22. P. Cortazar et al., "Pathological complete response and long-term clinical benefit in breast cancer: the CTNeoBC pooled analysis," Lancet 384(9938), 164-172 (2014).

23. C. Liedtke et al., "Response to neoadjuvant therapy and long-term survival in patients with triple-negative breast cancer," J. Clin. Oncol. 26(8), 1275-1281 (2008).

24. M. Untch et al., "Pathologic complete response after neoadjuvant chemotherapy plus trastuzumab predicts favorable survival in human epidermal growth factor receptor 2-overexpressing breast cancer: results from the TECHNO trial of the AGO and GBG study groups," J. Clin. Oncol. 29(25), 3351-3357 (2011).

25. S. K. Pal and A. Hurria, "Impact of age, sex, and comorbidity on cancer therapy and disease progression," J. Clin. Oncol. 28(26), 4086-4093 (2010).

26. G. S. Karagiannis et al., "Neoadjuvant chemotherapy induces breast cancer metastasis through a TMEM-mediated mechanism," Sci. Transl. Med. 9(397), eaan0026 (2017).

27. R. Rouzier et al., "Incidence and prognostic significance of complete axillary downstaging after primary chemotherapy in breast cancer patients with $\mathrm{T} 1$ to $\mathrm{T} 3$ tumors and cytologically proven axillary metastatic lymph nodes," J. Clin. Oncol. 20(5), 1304-1310 (2002).

28. D. R. McCready et al., "The prognostic significance of lymph node metastases after preoperative chemotherapy for locally advanced breast cancer," Arch. Surg. 124(1), 21-25 (1989).

29. N. Klauber-DeMore et al., "Size of residual lymph node metastasis after neoadjuvant chemotherapy in locally advanced breast cancer patients is prognostic," Ann. Surg. Oncol. 13(5), 685-691 (2006).

30. B. T. Hennessy et al., "Outcome after pathologic complete eradication of cytologically proven breast cancer axillary node metastases following primary chemotherapy," J. Clin. Oncol. 23(36), 9304-9311 (2005).

31. W. F. Symmans et al., "Measurement of residual breast cancer burden to predict survival after neoadjuvant chemotherapy," J. Clin. Oncol. 25(28), 4414-4422 (2007).

32. The University of Texas, "MD Anderson Cancer Center residual cancer burden calculator," http://www3.mdanderson.org/app/medcalc/index .cfm?pagename=jsconvert3 (2019). 
33. K. Burke, P. Tang, and E. Brown, "Second harmonic generation reveals matrix alterations during breast tumor progression," J. Biomed. Opt. 18(3), 031106 (2013).

34. W. S. Rasband, ImageJ, U.S. National Institutes of Health, Bethesda, Maryland (1997-2018) https://imagej.nih.gov/ij/.

35. C. A. Schneider, W. S. Rasband, and K. W. Eliceiri, "NIH Image to ImageJ: 25 years of image analysis," Nat. Methods $\mathbf{9}(7), 671-675$ (2012).

36. S. W. Perry et al., "Stromal matrix metalloprotease-13 knockout alters collagen I structure at the tumor-host interface and increases lung metastasis of C57BL/6 syngeneic E0771 mammary tumor cells," BMC Cancer 13, 411 (2013).

37. R. Rezakhaniha et al., "Experimental investigation of collagen waviness and orientation in the arterial adventitia using confocal laser scanning microscopy," Biomech. Model Mechanobiol. 11(3-4), 461-473 (2012).

38. D. A. Senthebane et al., "The role of tumor microenvironment in chemoresistance: to survive, keep your enemies closer," Int. J. Mol. Sci. 18(7), 1586 (2017).

39. S. Y. Sung et al., "Tumor microenvironment promotes cancer progression, metastasis, and therapeutic resistance," Curr. Probl. Cancer 31(2), 36-100 (2007).

40. O. D. Iseri et al., "Gene expression analysis of drug-resistant MCF-7 cells: implications for relation to extracellular matrix proteins," Cancer Chemother. Pharmacol. 65(3), 447-455 (2010).

41. A. L. Correia and M. J. Bissell, "The tumor microenvironment is a dominant force in multidrug resistance," Drug Resist. Updat. 15(1-2), 39-49 (2012).

42. Y. Brown, S. Hua, and P. S. Tanwar, "Extracellular matrix-mediated regulation of cancer stem cells and chemoresistance," Int. J. Biochem. Cell Biol. 109, 90-104 (2019).
43. R. K. Jain, "Delivery of molecular and cellular medicine to solid tumors," Adv. Drug Deliv. Rev. 46(1-3), 149-168 (2001).

44. E. B. Brown et al., "In vivo measurement of gene expression, angiogenesis and physiological function in tumors using multiphoton laser scanning microscopy," Nat. Med. 7(7), 864-868 (2001).

45. G. Alexandrakis et al., "Two-photon fluorescence correlation microscopy reveals the two-phase nature of transport in tumors," Nat. Med. 10(2), 203-207 (2004).

46. E. B. Brown et al., "Measurement of molecular diffusion in solution by multiphoton fluorescence photobleaching recovery," Biophys. J. 77(5), 2837-2849 (1999).

47. V. P. Chauhan et al., "Multiscale measurements distinguish cellular and interstitial hindrances to diffusion in vivo," Biophys. J. 97(1), 330-336 (2009).

48. V. P. Chauhan et al., "Delivery of molecular and nanoscale medicine to tumors: transport barriers and strategies," Апnи. Rev. Chem. Biomol. Eng. 2, 281-298 (2011).

49. S. Ramanujan et al., "Diffusion and convection in collagen gels: implications for transport in the tumor interstitium," Biophys. J. 83(3), 1650-1660 (2002).

50. T. Stylianopoulos et al., "Diffusion anisotropy in collagen gels and tumors: the effect of fiber network orientation," Biophys. J. 99(10), 3119-3128 (2010).

51. E. Brown et al., "Dynamic imaging of collagen and its modulation in tumors in vivo using second-harmonic generation," Nat. Med. 9(6), 796-800 (2003).

Biographies of the authors are not available. 